\title{
Aspectos cognitivos relacionados à noção de intervalos de tempo
}

\author{
Cognitive aspects related to time interval notion \\ Liliane Cristina de Além-Mar e Silva', Carla Cristina Adda²
}

\section{RESUMO}

Objetivo: Procuramos analisar os aspectos cognitivos e metodológicos envolvidos na avaliação da noção de intervalos de tempo, verificando os aspectos cognitivos envolvidos durante a sua execução, e apontar a relevância, aplicabilidade e sensibilidade dessa avaliação no exame neuropsicológico. Métodos: Realizou-se revisão bibliográfica abrangendo artigos e pesquisas das literaturas nacional e internacional no período de 1990 a 2005. Resultados: Encontraram-se 13 diferentes estudos. São controversas as discussões acerca de teorias sobre a noção de tempo. Os estudos podem ser agrupados de acordo com duas perspectivas diferentes: a teoria da contagem atencional, de grande utilização por pesquisadores americanos, e a teoria do relógio interno, muito abordada por estudiosos

\section{Palavras-chaves}

Neuropsicologia, funções executivas, estimativa de tempo, avaliação cognitiva.

\section{Key-words}

Neuropsychology,

executive functions, time estimation, cognitive evaluation. franceses que se diferem tanto em relação à abordagem teórica quanto à metodológica. Conclusões: Ambos os grupos de pesquisa, americanos e franceses, retratam a relevância da aplicação dos testes de estimativa, reprodução e produção de tempo no estudo das funções executivas.

\begin{abstract}
Objective: We tried to analyze the cognitive and methodological aspects involved in the evaluation of time intervals notion, verifying the cognitive aspects involved during your execution, and to point the relevance, applicability and sensibility of this evaluation in the neuropsychologic exam. Methods: Bibliographical revision was accomplished including articles and researches of the national and international literature in the period of 1990 to 2005. Results: Were found 13 different researches. The discussions concerning theories about the time notion are controversial. These studies can be grouped into two different perspectives: the attentional counter theory, used by American researchers, and the internal clock theory, very boarded by French studious, that are differed in relation to the theoretical and to the methodological approaches. Conclusions: Both research groups, Americans and French, show the relevance of the application of the timeinterval estimation tests, reproduction and production in the study of the executive functions.
\end{abstract}

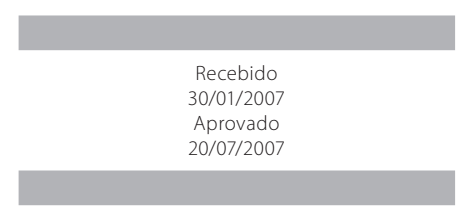

1 Divisão de Psicologia do Instituto Central do Hospital das Clínicas da Faculdade de Medicina da Universidade de São Paulo (DIP/ICHC-FMUSP). 2 DIP/ICHC-FMUSP e Departamento de Neurologia do ICHC-FMUSP.

Endereço para correspondência: Liliane Cristina de Além-Mar e Silva Rua Dona Elvira Andrade Cunha, 310 - 38080-430 - Uberaba, MG Fone: (34) 3338-8354. E-mail: lili_psico@hotmail.com 


\section{INTRODUÇÃO}

O tempo é uma informação essencial para a estruturação de nosso comportamento, e o processamento dessa informação depende de múltiplos processos cognitivos, como a inibição de distratores. As funções executivas se referem a uma variedade de habilidades que incluem iniciação, planejamento, desenvolvimento de estratégias, persistência, flexibilidade, controle inibitório, memórias operacionais verbal e não-verbal, auto-regulação e velocidade de processamento mental (Lezak, 1995). Essas habilidades são alvo de estudos teóricos e empíricos de populações que conhecidamente encontram dificuldades em algum aspecto do funcionamento executivo, como pessoas com transtorno de déficit de atenção e com lesões na região frontal do córtex cerebral (Barkley et al., 2000). A noção de intervalos de tempo é um processo do funcionamento cognitivo que vem sendo amplamente estudado, sugerindo uma nova modalidade de investigação neuropsicológica para o estudo das funções executivas.

Muitos estudos (Barkley et al., 2000; 2001; 2005) têm sido desenvolvidos sobre funções executivas em pessoas com transtorno de déficit de atenção com hiperatividade (TDAH), e são variados e numerosos os déficits encontrados. O TDAH é caracterizado pelo desenvolvimento inapropriado e prejudicado dos níveis atencionais, com a presença de comportamento hiperativo-impulsivo ou ambos que surgem na infância (Associação Americana de Psiquiatria, 1994). Uma área de déficits cognitivos associados ao TDAH é a noção de tempo.

Para avaliar a noção de tempo, desenvolveram-se vários métodos, e os três mais comumente utilizados são os de estimativa, produção e reprodução (Zakay, 1990). No teste de estimativa, o examinando deve expressar verbalmente quanto tempo certo intervalo durou. No teste de produção, o examinador diz um intervalo específico e o examinando deve produzi-lo de alguma forma, ligando e desligando uma lanterna, por exemplo. Na reprodução de tempo, demonstra-se para o examinando uma duração e este deve replicála. Nesse método, ele deve estar atento a duas condições: à passagem do tempo, realizando comparações com outras formas de medida temporal, como acontece na estimativa; à reprodução motora do intervalo. A reprodução parece ser o teste mais complexo, pois conta com maior demanda atencional ou memória de trabalho para o processamento de tempo (Brown, 1990; Mimura et al., 2000; Zakay, 1990).

Avaliada por meio do teste de reprodução de tempo, a inibição motora associa-se à memória de trabalho nãoverbal, sem a qual a pessoa não é capaz de manter medidas de tempo para associá-las a eventos que devem ser seqüencialmente distribuídos em um intervalo (Brown, 1990). No entanto, outros aspectos cognitivos também es- tão envolvidos. Pesquisas demonstram que a memória de trabalho é importante para a execução exata de intervalos de tempo de 1 segundo ou mais de duração (Mimura et al., 2000), enquanto a percepção de tempo envolvendo intervalos menores que 1 segundo é mediada pelos gânglios da base (Harrington et al., 1998) e cerebelo (Mangels et al., 1998). Para intervalos maiores que 20 a 30 segundos, também são envolvidas a memória episódica ou a memória de longo prazo, já que essas durações excedem o campo da memória de curto prazo e da memória de trabalho (Brown, 1990; Kinsbourne e Hicks, 1990; Mimura et al., 2000). Por meio de vários estudos americanos realizados com pessoas com TDAH, indica-se que elas não obtêm desempenho inferior em testes de estimativa de tempo. No entanto, são conhecidos os resultados deficitários em reprodução do tempo percebido (Barkley et al., 2001).

A avaliação da noção de tempo é amplamente conhecida entre estudiosos do TDAH, porém é uma modalidade de avaliação ainda pouco estudada e utilizada por neuropsicólogos na investigação do funcionamento executivo de seus pacientes. É possível que outros tipos de enfermidades que acometem o funcionamento cognitivo obtenham como conseqüência a capacidade inapropriada de estimar, produzir e/ou reproduzir intervalos de tempo. Isto sugere a necessidade de ampliação de estudos referentes a esse método de investigação para outros campos de atuação da neuropsicologia. O objetivo do presente artigo foi realizar uma revisão bibliográfica sobre aspectos cognitivos e metodológicos envolvidos na avaliação da noção de intervalos de tempo. Segundo essa idéia geral, também buscamos verificar, por meio da literatura revisada, a utilização dos recursos de avaliação da noção de intervalos de tempo na prática neuropsicológica; descrever os aspectos cognitivos envolvidos durante a execução da estimativa e da reprodução de intervalos de tempo; e analisar e descrever a relevância, aplicabilidade e sensibilidade do teste de estimativa e de reprodução de intervalos de tempo na avaliação neuropsicológica.

\section{MÉTODOS}

Revisão das literaturas nacional e internacional sobre estimativa e reprodução de intervalos de tempo em revistas e artigos indexados nas bases de dados Pubmed, Lilacs e Portal Periódicos Capes/Banco de dados brasileiro de teses e dissertações (PPC), publicados no período de 1990 a 2006. Utilizou-se tal período na tentativa de encontrar maior quantidade de artigos sobre o assunto, visto ser um tema pouco abordado em estudos neuropsicológicos. Utilizaram-se como palavras-chave os termos "estimativa de tempo", "reprodução de tempo" e "funções executivas". 
Além das referências localizadas nas bases de dados citadas, autores e textos relacionados apontados pelo Pubmed também foram considerados na procura por maior número de estudos sobre o tema. Na busca por meio da Lilacs, consideraram-se apenas resumos nas línguas portuguesa e inglesa. Os critérios seguidos para o levantamento bibliográfico foram: artigos com resumo; clara menção de estudos metodológicos em noção de intervalos de tempo no corpo do resumo.

\section{RESULTADOS}

Encontraram-se e consideraram-se 13 diferentes estudos. Destes, 11 são descritos na tabela 1. Os outros dois trabaIhos apenas descrevem a noção de intervalos de tempo, não apontando informações nem vínculos teóricos sobre os paradigmas analisados neste artigo. São controversas as discussões acerca de teorias sobre a noção de tempo. Observam-se duas diferentes opiniões sobre o tema: a teoria da contagem atencional, de grande utilização por pesquisadores americanos, e a teoria do relógio interno, muito abordada por estudiosos franceses (Hick et al., 1997).

\section{DISCUSSÃO}

A primeira teoria descreve que há um relógio cognitivo que efetua contagens de eventos temporais, e essa contagem é diretamente dependente da atenção (Zakay, 1993). Diversas pesquisas no campo da psicologia comportamental demonstraram que o fraco controle inibitório se associa a dificuldades na noção temporal, mais particularmente em paradigmas de reprodução de tempo (Pavlov, 1927).

De acordo com essa teoria, quando submetidas a atividades que necessitam da estimativa e reprodução de intervalos de tempo, pessoas com menos habilidade para inibir interferências tendem a cometer maior número de erros do que pessoas com maior habilidade. Deve haver muitas razões para essa relação, como, por exemplo, aspectos atencionais, que são descritos como importantes na percepção prospectiva do tempo em crianças e adultos (Zakay, 1992).

Para a teoria da contagem atencional, a noção de tempo é vista como o funcionamento direto da total atenção destinada à passagem do tempo (Zakay, 1992). Contando com o funcionamento dos sistemas atencionais e de memória de trabalho, quanto mais um indivíduo destina atenção para informações não-temporais, menos ele a destina para processos temporais e, então, menor parece ser sua noção de passagem do tempo. Quanto mais atenção é deslocada para processamentos temporais, maior parecerá ser a duração do tempo em questão. Interferências podem ocorrer durante a passagem do tempo e serão alvo de atenção para informações não-temporais.

Nesse caso, muitos erros são esperados na percepção do tempo (Zakay, 1992). Tudo isso sugere que pessoas impulsivas, que encontram relativa dificuldade com distrações e atenção, devem manifestar maiores dificuldades na noção de tempo do que pessoas não impulsivas (Barkley, 2001).

De acordo com a segunda teoria citada, a do relógio interno, a estimativa de tempo envolve um sistema neural, e o cérebro destina diversas áreas para a devida implementação da noção temporal. A idéia geral é que um certo estímulo pode sincronizar neurônios em uma área do córtex, que atua como um sinal inicial. Como cada neurônio produz seu padrão particular de ativação durante todo o tempo, cada intervalo se associa a um único padrão de ativação, e todos esses padrões são interpretados por outras regiões cerebrais nos gânglios basais (Matell e Meck, 2000).

Ao contrário da teoria da contagem atencional, o sistema de contagem de tempo na teoria do relógio interno não requer atenção e envolve três etapas: acumulação de unidades temporais, armazenamento, comparação e decisão (Church, 1984). Na acumulação de unidades temporais, um marcapasso emite pulsos constantes, e esses pulsos são mantidos em um acumulador, onde fica armazenado todo material da medição de tempo. Dependendo da amplitude do intervalo de tempo, esse armazenamento se refere à memória de trabalho ou à memória de longo prazo. Finalmente, ocorre um mecanismo de comparação dos valores de duração de tempo observado do meio externo com aquele internamente armazenado na memória. Em resultado a essa comparação, a pessoa toma a decisão da adequada resposta temporal (Church, 1984).

Apesar de a teoria do relógio interno não incorporar a idéia atencional elaborada pela teoria da contagem atencional, esta última descreve as etapas de armazenamento, comparação e decisão (Taatgen et al., 2003). Tal fato confirma o envolvimento direto das funções cognitivas no processo de noção temporal, tanto na etapa de estimativa de tempo quanto nas de produção e reprodução de intervalos de tempo, independentemente da teoria escolhida.

Também são notórias as diferenças metodológicas entre estudiosos da noção de intervalos de tempo. Autores americanos (Barkley et al., 2000; 2001; 2005) têm desenvolvido estudos utilizando quantidades maiores de intervalos de tempo durante a testagem, totalizando seis intervalos superiores a 2 segundos, que são expostos ao sujeito em duas ordens diferentes para tarefas de estimativa e de reprodução de tempo. Dessa forma, utilizando uma lanterna que deve ser ligada para demarcar início do intervalo e desligada a fim de finalizar o mesmo intervalo, o examinando deve estimar seis intervalos. Em seguida, são expostos os mesmos intervalos em uma ordem diferente da primeira. Realizada em seguida à estimativa, a etapa da reprodução 
de tempo ocorre da mesma forma: o indivíduo utiliza uma lanterna idêntica à do examinador para reproduzir seis intervalos que são expostos duas vezes, em dois tipos de ordenamentos.

Autores franceses (Pouthas et al., 2003; Pouthas e Perbal, 2004) descrevem a utilização de computadores para as etapas de reprodução e produção de intervalos de tempo, respectivamente. Utilizam-se três intervalos de tempo maiores que 2 segundos, nos quais se mostra ao examinando um quadrado azul na tela do monitor. Após aparecer a primeira imagem, o sujeito deve apertar um botão que aciona o aparecimento do quadrado e, ao decidir que o intervalo desejado foi atingido, o botão novamente deve ser acionado e o quadrado desaparece. Esses mesmos três intervalos são expostos em cinco diferentes ordens randomicamente distribuídas. Em seguida, realiza-se a produção de intervalos de tempo, na qual o examinando aciona o botão para aparecimento do quadrado logo após o examinador dizer o tempo que deve ser produzido e, então, o aciona novamente para o desaparecimento deste, assim que achar que o intervalo cessou. Enfim, os mesmos três intervalos são requisitados para o indivíduo cinco vezes, em cinco ordens diferentes.

Em ambos os paradigmas explicitados, intervalos aleatórios de treino são propostos antes da execução das diferentes etapas da testagem, para que o examinador possa estar certo da compreensão do examinando.

Autores que se baseiam na teoria da contagem atencional têm utilizado os testes de estimativa e reprodução de tempo a fim de conhecer as limitações e potencialidades de pacientes portadores de TDAH em todas as faixas etárias (Barkley et al., 2000; 2001; 2005). Enquanto isso, estudiosos da teoria do relógio interno têm expandido recentemente o estudo da noção temporal através de populações clínicas mais abrangentes, como adultos maduros, portadores de doença de Parkinson, pessoas com traumatismos corticais agudos, pacientes amnésicos e pacientes que sofreram ressecções de lobo temporal mesial (Pouthas e Perbal, 2004; Pouthas et al., 2005). Para sobreposição de dados, cada um dos grupos citados complementou as testagens de noção temporal com diferentes instrumentos de reconhecimento científico (tabela 1).

A partir do pressuposto teórico da contagem atencional, os pesquisadores concluíram que pessoas com TDAH obtêm resultados semelhantes aos controles nos testes de estimativa de tempo, que avaliam principalmente memória de trabalho ou memória de longo prazo. No entanto, quando submetidos à reprodução de tempo, esses pacientes obtêm resultados deficitários em virtude do mau funcionamento atencional, influenciado pela baixa capacidade de inibir distratores internos e externos (Barkley et al., 2001). Assim, os métodos de avaliação temporal indicam preservação da capacidade de memorização dos pacientes com
TDAH e dificuldade nos processos competentes às funções executivas, tais como controle inibitório e atenção.

Algumas limitações e críticas aos seus próprios estudos são apontadas pelo grupo que estuda a teoria da contagem atencional. Entre elas, a ordem de aplicação dos métodos, primeira estimativa e, em seguida, a reprodução de intervalos de tempo poderiam influenciar mais no pior desempenho das crianças com TDAH na reprodução do que na estimativa de tempo (Barkley et al., 2001). Apesar de o autor admitir que essa ordem poderia diminuir o desempenho dos sujeitos, por estarem exaustos no momento da aplicação da reprodução de intervalos de tempo, afirma que seus resultados são consistentes com estudos anteriores com o mesmo perfil de sujeitos. Também se aponta que a modalidade computadorizada poderia sugerir maior precisão na apuração de resultados dos pacientes, em detrimento da avaliação manual com a utilização de estímulos e cronômetros manuais (Barkley et al., 2001).

Nas recentes publicações com a utilização da reprodução e produção de intervalos de tempo, pesquisadores da abordagem do relógio interno têm encontrado indicativos de relação entre a noção temporal e os aspectos cognitivos: velocidade de processamento e memória (Pouthas e Perbal, 2004). No entanto, no mesmo estudo se expõe a ligação de seus achados com o seu público-alvo, o qual apresenta proeminentes problemas com a memória. O aprofundamento dos estudos das relações entre julgamento de duração de intervalos e funções executivas, utilizando-se de vários grupos de pacientes, é sugerido pelos próprios autores.

A avaliação da noção de intervalos de tempo, apesar de se basear em estudos desenvolvidos e estudados há tempos, é pouco conhecida por profissionais da neuropsicologia e estudiosos das funções executivas. Muitos dos trabalhos realizados na área têm desenvolvido diferentes paradigmas para a melhor compreensão dos mecanismos neurocognitivos envolvidos na noção de tempo.

São comumente utilizados três diferentes métodos para avaliar de forma direta a noção de tempo: estimativa, produção e reprodução. Com a finalidade de investigar pacientes caracterizados pela desatenção e dificuldades em inibir distratores, estudiosos da teoria da contagem atencional optaram por utilizar os métodos de estimativa e reprodução de tempo. Concluíram que os sujeitos obtêm desempenho normal em estimativa e subnormal em reprodução de tempo e certificaram-se de que as dificuldades atencionais e inibição são proeminentes nesse público-alvo em comparação aos problemas com memória. Investigando pessoas previamente conhecidas por obterem baixa performance em avaliações da memória, o grupo de estudiosos da teoria do relógio interno optou por utilizar os métodos de produção e reprodução de intervalos de tempo. Parece que seria prolixa a aplicação da estimativa de tempo neste público, dado seu direcionamento principal para a avaliação mnemônica. 


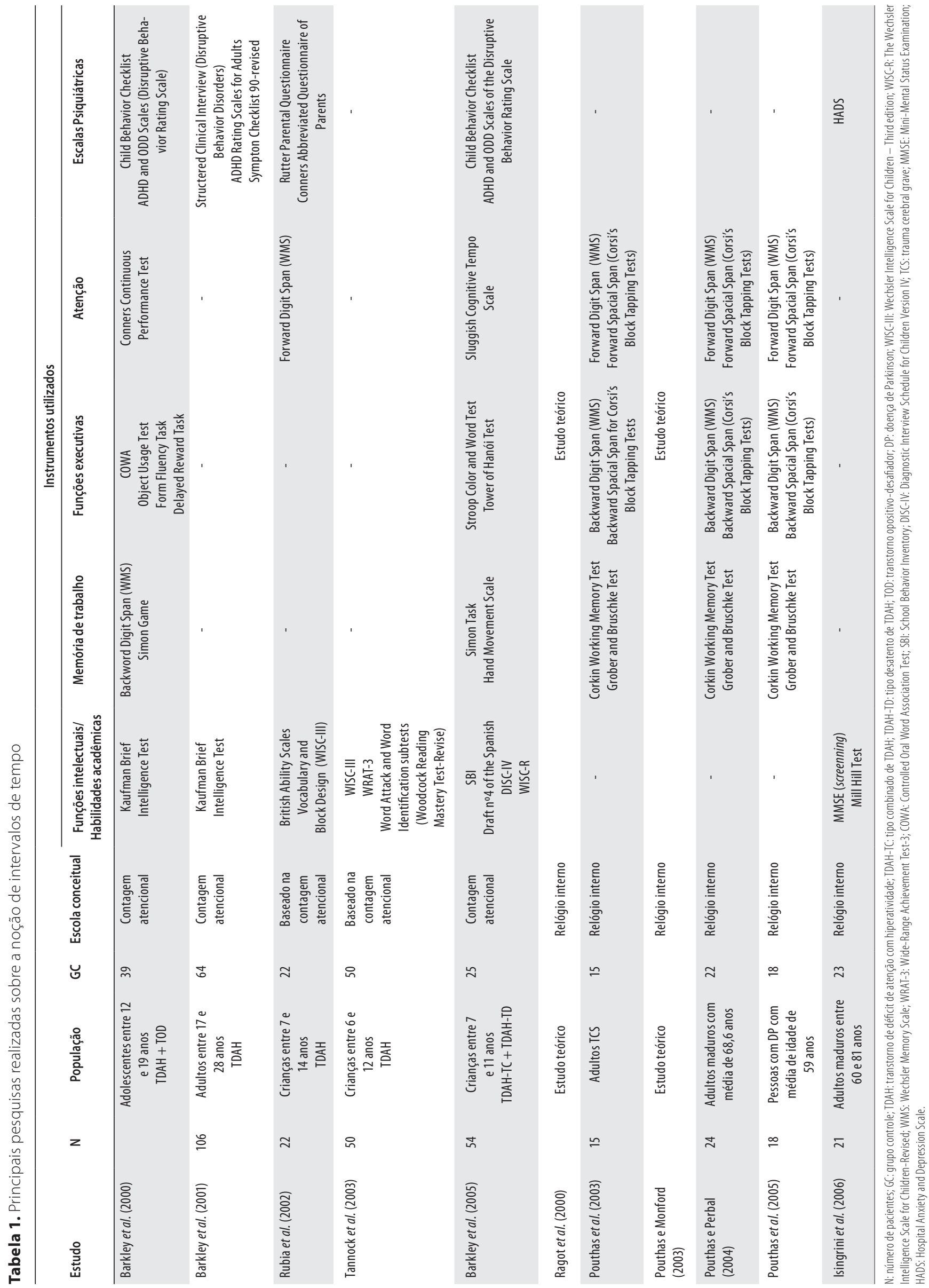


Em função de seus achados, os métodos de produção e reprodução de intervalos de tempo são indicados para a investigação de outro importante aspecto das funções executivas, a velocidade de processamento de informações. Portanto, da mesma forma como o neuropsicólogo efetua a escolha dos testes a serem incluídos em uma bateria, cada um desses métodos deve ser utilizado de acordo com a função cognitiva de necessária investigação.

Para respaldar os dados obtidos nas avaliações de noção temporal, cada grupo de estudiosos compôs baterias diferentes que evidenciam, em um caso, as funções executivas e, no outro, a memória e velocidade de processamento. As diferenças metodológicas apontadas anteriormente também são indicativas da opção dos autores em função do público a ser investigado.

A fim de verificar em que ponto as pessoas com TDAH passam a tolerar menos as interferências e se tornam desatentas, cientistas do paradigma da contagem atencional optaram pela exposição de seis diferentes intervalos de tempo maiores que 2 segundos. Assim, os mesmos intervalos são repetidos apenas duas vezes.

Para investigar a consistência de respostas de seu grupo, estudiosos da teoria do relógio interno escolheram submeter os sujeitos a apenas três intervalos de tempo superiores a 2 segundos. No entanto, o foco de seu estudo está na repetição desses três intervalos, já que o importante para seus objetivos passa a ser a coerência ou não de respostas do sujeito.

Os resultados desses estudos demonstram a sensibilidade dos métodos de avaliação da noção de tempo e demonstram aos estudiosos das funções executivas um novo campo de investigação. Nesse sentido, encontrou-se um pequeno número de trabalhos sobre a noção de intervalos de tempo, e nenhum deles realizou a comparação entre os paradigmas francês e americano. Tal fato possivelmente se deve à dificuldade em comparar trabalhos com metodologias diferentes desde o material utilizado até o público investigado (em idade e morbidade neurológica).

De acordo com suas necessidades e possibilidades estruturais, neuropsicólogos podem encontrar nos métodos de exame da noção temporal importantes instrumentos para a confirmação de resultados de outros testes e/ou complementação da avaliação do funcionamento executivo de seus pacientes ou sujeitos de pesquisas.

\section{CONCLUSÕES}

Independentemente do paradigma e da metodologia adotados pelos estudos descritos, ambos apontam importantes caminhos para o acesso a aspectos das funções executivas e sugerem a avaliação de algo ainda pouco abordado no exame neuropsicológico clínico: a noção de intervalos de tempo.
Como indicado pelos autores das teorias da contagem atencional e do relógio interno, o aprofundamento dos estudos de diferentes populações clínicas utilizando os métodos de estimativa, produção e reprodução é de grande necessidade. No entanto, mesmo os estudos já existentes não tratam de investigações de populações culturalmente compatíveis com a população brasileira, o que indica a importância da discussão de pesquisadores brasileiros acerca das teorias aqui expostas. Sugerimos que esse meio eficaz de análise das funções executivas faça parte de baterias neuropsicológicas a serem utilizadas em várias idades e contextos da neuropsicologia clínica e científica.

\section{REFERÊNCIAS}

American Psychiatric Association. Diagnostic and statistical manual of mental disorders. 4th ed. Washington DC, 1994.

Barkley RA, Murphy KR, Bush T. Time perception and reproduction in young adults with attention deficit hyperactivity disorder. Neuropsychology, 15:351-60, 2001.

Barkley RA et al. Executive functioning, temporal discounting, and sense of time in adolescents with attention deficit hyperactivity disorder (ADHD) and oppositional defiant disorder (ODD). Journal of Abnormal Child Psychology, 29(6):541-56, 2000.

Barkley RA et al. Time estimation and performance on reproduction tasks in subtypes of children with attention deficit hyperactivity disorder. Journal of Clinical Child and Adolescent Psychology, 34(1):151-62, 2005

Brown JW. Psychology as time awareness. Brain and Cognition, 14:144-64, 1990.

Church RM. Properties of the internal clock. In: Gibbon J, Allan L. Timing and perception. Ann NY Acad Sci, 423:566-82, 1984.

Harrington DL, Haaland KY, Hermanowics N. Temporal processing in the basal ganglia. Neuropsychology, 12:3-12, 1998.

Hick ER et al. Concurrent processing demands and the experience of time in passing. American Journal of Psychology, 90:413-46, 1997

Isingrini M et al. Age-related changes in duration reproduction: Involvement of working memory processes. Brain and Cognition, 62:17-23, 2006

Kinsbourne M, Hicks RE. The extended present: evidence from time estimation by amnesics and normals. In: Vallar G, Shallice T, eds. Neuropsychological impairments of short-term memory. Cambridge: Cambridge University Press p. 319-29, 1990

Lezak MD. Neuropsychological assessment. 3. ed. New York: Oxford University Press; 1995.

Mangels JA, Ivry RB, Shimizu N. Dissociable contributions of the prefrontal and neocerebellar cortex to time perception. Cognitive Brain Research, 7:15-39, 1998.

Matell MS, Meck WH. Neuropsychological mechanisms of interval timing behavior. BioEssays, 22:94-103, 2000

Mimura M, Kinsbourne M, O'Connor M. Time estimation by pacients with frontal lesions and by Korsakoff amnesics. Journal of Neuropsychological Society, 6:517-28, 2000.

Pavlov IP. Conditioned reflexes. London: Oxford University Press; 1927.

Pouthas V, Monford V. Effects of working memory demands on frontal slow waves in timeinterval reproduction tasks in humans. Neuroscience Letters, 343:195-9, 2003.

Pouthas V, Perbal S. Time perception depends on accurate clock mechanisms as well as unimpaired attention and memory process. Acta Neurobiologiae Experimentalis, 64:367-85, 2004.

Pouthas V et al. Relationships between time estimation, memory, attention, and processing speed in pacients with several traumatic brain injury. Neuropsychologia, 41:1599-610, 2003.

Pouthas $V$ et al. Effects of internal clock and memory disorders on duration reproductions and duration productions in pacients with Parkinson's disease. Brain and Cognition, 58:35-48, 2005.

Ragot R, Monfort V, Pouthas V. Role of frontal cortex in memory for duration: an event-related patient study in humans. Neuroscience Letters, 286:91-4, 2000. 
Rubia Ket al. Evidence for a pure time perception deficit in children with ADHD. Journal of Child Psychology an Psychiatry, 43 (4):529-42, 2002.

Taatgen $\mathrm{N}$ et al. Time interval estimation: internal clock or attentional mechanism? Human Factors, 45(1):61-76, 2003.

Tannock R et al. Time perception deficits in attention-deficit/hyperactivity disorder and comorbid reading difficulties in child and adolescent samples. Journal of Child Psychology and Psychiatry, 44(6):888-903, 2003.
Zakay D. The evasive art of subjective time measurement: some methodological dilemmas. In: Block RA, ed. Cognitive models of psychological time. Hillsdale: Lawrence Erbaum Associates; 1990, p. 59-84.

Zakay D. The role of attention in children's time perception. Journal of experimental child psychology, 54:355-71, 1992

Zakay D. Time estimation methods-do they influence prospective duration estimates? Perception, 22:91-101, 1993 